\title{
Diabetic Ketoacidosis at Emergency Department Presentation During the First Months of the SARS- CoV-2 Pandemic in Israel: A Multicenter Cross- Sectional Study
}

\author{
Ron Jacob · Giora Weiser · Danna Krupik · Dania Takagi · Shuny Peled • Naama Pines · \\ Saar Hashavya · Hagar Gur-Soferman · Shirly Gamsu • Or Kaplan • Michal Maimon · Shahar Oren • \\ Gabi Padeh · Itai Shavit (D)
}

Received: January 26, 2021 / Accepted: March 4, 2021 / Published online: March 17, 2021

(c) The Author(s) 2021

\section{ABSTRACT}

Introduction: We aimed to examine the impact of the severe acute respiratory syndrome coronavirus 2 (SARS-CoV-2) pandemic on diabetic ketoacidosis (DKA) rates in children with type 1 diabetes (T1D).

Methods: A retrospective cross-sectional study of 11 Israeli pediatric emergency departments (ED) was conducted. Children with T1D who

\section{R. Jacob}

Pediatric Emergency Department, Ha'Emek Medical Center, Afula, Israel

\section{G. Weiser}

Pediatric Emergency Department, Shaare Zedek

Medical Center, Jerusalem, Israel

D. Krupik

Pediatric Emergency Department, Ziv Medical

Center, Tzfat, Israel

\section{Takagi}

Pediatric Emergency Department, Meir Medical

Center, Kfar Saba, Israel

S. Peled · I. Shavit $(\bowtie)$

Pediatric Emergency Department, Rambam Health

Care Campus, Haifa, Israel

e-mail: itai@pem-database.org

N. Pines $\cdot$ S. Hashavya

Pediatric Emergency Department, Hadassah Medical

Center, Jerusalem, Israel attended the ED between March 1, 2020 and May 31, 2020 were compared with those who attended the ED between March 1, 2019 and May 31, 2019.

Results: Overall, 150 and 154 children with T1D attended the EDs during the 3-month study periods in 2020 and 2019, respectively. Among patients with established T1D, DKA rates significantly increased in 2020 compared to $2019 \quad[38 / 64(59.3 \%)$ vs $31 / 74 \quad(41.9 \%)$; $p<0.043]$. There was a non-statistically significant trend toward a higher rate of DKA in

H. Gur-Soferman

Pediatric Emergency Department, Samson Assuta Ashdod University Hospital, Ashdod, Israel

\section{S. Gamsu}

Pediatric Emergency Department, Shamir Medical Center, Beer Yaakov, Israel

\section{O. Kaplan · M. Maimon}

Pediatric Emergency Department, Soroka Medical Center, Beer Sheba, Israel

\section{S. Oren}

Pediatric Emergency Department, Kaplan Medical Center, Rehovot, Israel

G. Padeh

Emergency Department, Schneider Children's Medical Centre, Petah Tikva, Israel 
patients with newly diagnosed T1D [46/86 $(53.4 \%)$ vs $31 / 80$ (38.7\%); $p=0.063$. No differences were observed in the rates of severe DKA in 2020 compared to 2019 among patients with established T1D [10/64 (15.6\%) vs 6/74 $(8.1 \%) ; p=0.184]$, and newly diagnosed T1D [16/86 (18.6\%) vs $14 / 80(17.5 \%) ; p=0.858]$. No differences were observed in the rates of intensive care unit admissions in 2020 compared to 2019 among patients with established T1D [14/ $64(21.8 \%)$ vs $14 / 74(18.9 \%) ; p=0.672]$, and newly diagnosed T1D [26/86 (30.2\%) vs $21 / 80$ $(26.2 \%) ; p=0.977]$.

Conclusions: Increased rates of DKA in children with established T1D were observed during the first 3 months of the outbreak in Israel. The findings suggest that the severity of DKA at ED presentation in children with T1D was not influenced by the pandemic.

Keywords: Coronavirus; Diabetes; Ketoacidosis; Pandemic

\section{Key Summary Points}

Why carry out this study?

Diabetic ketoacidosis (DKA) is a severe acute complication of diabetes which occurs more often in children with type 1 diabetes (T1D).

We examined DKA at emergency department presentation in patients with established and newly diagnosed T1D during the SARS-CoV-2 outbreak in Israel.

\section{What was learned from the study?}

We found that the rates of DKA significantly increased during the pandemic period among patients with established T1D.

There was a trend toward higher rates of DKA among patients with newly diagnosed T1D.

Since the pandemic is still progressing, it is important to enhance awareness of this phenomenon while simultaneously exploring possible causes.

\section{DIGITAL FEATURES}

This article is published with digital features, including a summary slide to facilitate understanding of the article. To view digital features for this article go to https://doi.org/10.6084/ m9.figshare.14153168.

\section{INTRODUCTION}

Diabetic ketoacidosis (DKA) is a severe acute complication of diabetes arising from a relative insulin deficiency, which occurs more often in children with type 1 diabetes (T1D) and in the setting of moderate to severe illness [1]. Recent studies found an increase in the rates of severe DKA in patients with newly diagnosed T1D during the severe acute respiratory coronavirus 2 (SARS-CoV-2) pandemic [2-4]. There have been no studies that examined the impact of the SARS-CoV-2 pandemic on DKA rates in emergency department (ED) patients with established T1D.

We examined DKA at ED presentation in patients with established T1D and newly diagnosed T1D (diagnosis at admission) during the first 3 months of the SARS-CoV-2 outbreak in Israel.

\section{METHODS}

\section{Study Design}

A retrospective cross-sectional study of 11 pediatric EDs was conducted. Children with T1D who attended the ED between March 1, 2020 and May 31, 2020 were compared with those who attended the ED between March 1, 2019 and May 31, 2019.

\section{Participant Selection and Data Source}

Only children who were coded as having a diabetes-related presentation as their reason for the visit were included. The following variables were abstracted: age, sex, type of T1D (newly diagnosed, established), presence of DKA, 
period of admission (pandemic, pre-pandemic), serum $\mathrm{pH}$ and bicarbonate levels on admission, and intensive care unit admission. Chart review was conducted in accordance with published methods for retrospective chart review studies in emergency medicine [5]. The study was approved by the institutional review boards of the participating hospitals, and was performed in accordance with the principles embodied in the Declaration of Helsinki. Informed consent was not required because of the retrospective nature of the study. DKA was defined as a $\mathrm{pH}$ level $<7.3$. Severe DKA was defined as a $\mathrm{pH}$ level $<7.1[6]$.

\section{Statistical Analysis}

Fisher's exact test was used to compare proportions between groups. The non-parametric Mann-Whitney $U$ test was used to compare continuous variables. A two-sided $p<0.05$ was considered statistically significant. All statistics were calculated using StatsDirect statistical software (V.2.6.6, StatsDirect Limited, Cheshire, UK).

\section{RESULTS}

Overall, 48,176 and 77,477 patients visited the pediatric EDs during the study periods in 2020 and 2019, respectively. One-hundred and fifty patients with T1D and a median age (interquartile range, IQR) of 12.0 (8.7-14.1) years, and 154 patients with T1D and a median (IQR) age of 12.0 (8.7-15.0) years attended the pediatric EDs during the 2020 and 2019 study periods, respectively.

There were 64 and 74 patients with established T1D in 2020 and 2019, respectively; and 86 and 80 patients with newly diagnosed T1D in 2020, and 2019, respectively (Table 1 ).

Distributions of DKA severity categories (mild, moderate, severe) are shown in Fig. 1.

Among patients with established T1D, the rate of DKA significantly increased in the pandemic period compared to the pre-pandemic period $[38 / 64 \quad(59.3 \%)$ vs $31 / 74 \quad(41.9 \%)$, $p<0.043]$, Table 1 . There was a non-statistically significant trend toward a higher rate of DKA in patients with newly diagnosed T1D [46/86 (53.4\%) vs 31/80 (38.7\%), $p=0.063]$, Table 1.

No differences were observed in the rates of severe DKA in 2020 compared to 2019 among patients with established T1D [10/64 (15.6\%) vs $6 / 74(8.1 \%) ; p=0.184]$, and newly diagnosed T1D $[16 / 86 \quad(18.6 \%)$ vs $14 / 80 \quad(17.5 \%)$; $p=0.858]$.

No differences were observed in the rates of intensive care unit admissions in 2020 compared to 2019 among patients with established T1D $[14 / 64 \quad(21.8 \%)$ vs $14 / 74 \quad(18.9 \%)$; $p=0.672]$, and newly diagnosed T1D [26/86 $(30.2 \%)$ vs $21 / 80(26.2 \%) ; p=0.977]$. None of the patients died.

Thirteen/86 (15.1\%) patients with newly diagnosed T1D visited the ED during the lockdown period (March 17, 2020 to April 17, 2020), 9/13 (69.2\%) had DKA at presentation. Thirteen/64 (20.3\%) patients with established T1D visited the ED during the lockdown period, 9/13 (69.2\%) had DKA at presentation.

\section{DISCUSSION}

This study reveals a $37.8 \%$ reduction in pediatric ED visits during the first 3 months of the pandemic in Israel. Parents and caregivers' perception of the ED as a place of infection is maybe the cause of the decrease in ED utilization [7].

The main finding of the current study is the increased rate of DKA in children with established T1D who attended the ED because of a diabetes-related problem during the first 3 months of the pandemic period. The lower serum $\mathrm{pH}$ and bicarbonate levels at ED presentation during the pandemic period compared with the pre-pandemic period support this finding (Table 1).

Another important finding is that there were no differences in the rate of severe DKA at ED presentation (Table 1) and in the rate of intensive care unit admissions due to DKA between the two study periods. These findings suggest that the severity of DKA at ED presentation of patients with T1D was not influenced by the pandemic. 
Table 1 Comparison of patients with newly diagnosed and established type 1 diabetes during the pandemic (March 1-May 31, 2020) and pre-pandemic (March 1-May 31, 2019) periods

\begin{tabular}{|c|c|c|c|}
\hline & Pandemic period $(n=86)$ & Pre-pandemic period $(n=80)$ & $p$ value \\
\hline \multicolumn{4}{|l|}{ Newly diagnosed type 1 diabetes } \\
\hline Patients with $\mathrm{DKA}^{\mathrm{a}}, n(\%)$ & $46(53.4)$ & $31(38.7)$ & 0.063 \\
\hline Patients with severe $\mathrm{DKA}^{\mathrm{b}}, n(\%)$ & $16(18.6)$ & $14(17.5)$ & 0.858 \\
\hline Serum pH level, mean (SD) & $7.24(0.16)$ & $7.26(0.17)$ & 0.142 \\
\hline \multirow[t]{2}{*}{ Serum bicarbonate level, mmol/L, mean (SD } & ) $16.3(7.8)$ & $18.1(8.6)$ & 0.091 \\
\hline & Pandemic period $(n=64)$ & Pre-pandemic period $(n=74)$ & $p$ value \\
\hline \multicolumn{4}{|l|}{ Established type 1 diabetes } \\
\hline Patients with $\mathrm{DKA}^{\mathrm{a}}, n(\%)$ & $38(59.3)$ & $31(41.9)$ & 0.043 \\
\hline Patients with severe $\mathrm{DKA}^{\mathrm{b}}, n(\%)$ & $10(15.6)$ & $6(8.1)$ & 0.184 \\
\hline pH level, mean (SD) & $7.24(0.13)$ & $7.29(0.11)$ & 0.015 \\
\hline Bicarbonate level, mmol/L, mean (SD) & $16.3(7.5)$ & $19.0(7.7)$ & 0.041 \\
\hline
\end{tabular}

$S D$ standard deviation, $D K A$ diabetic ketoacidosis

a Diabetic ketoacidosis: $\mathrm{pH}$ level $<7.3$

b Severe diabetic ketoacidosis: $\mathrm{pH}$ level $<7.1$

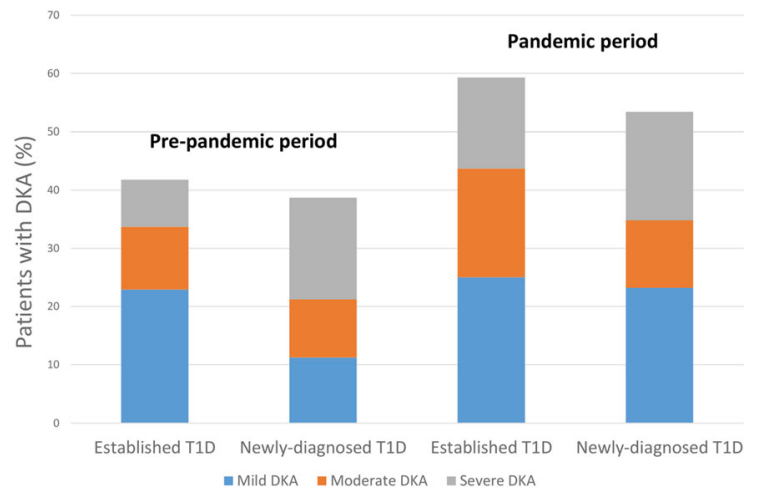

Fig. 1 Rates of diabetic ketoacidosis (DKA) in patients with newly diagnosed and established type 1 diabetes on emergency department admission during the pandemic (March 1-May 31, 2020) and pre-pandemic (March 1-May 31, 2019) periods. Definitions of DKA severity [6]: DKA, pH level $<7.3$; mild DKA, $7.2<\mathrm{pH}$ level < 7.3; moderate DKA, $7.1<\mathrm{pH}$ level $<7.2$; severe DKA, pH level $<7.1$

Although not statistically significant, there seems to be a trend toward a higher rate of DKA during the pandemic period in patients with newly diagnosed T1D as well (53.4\% vs 38.7\%, Table 1). This finding was also reported in recent studies from Germany, Italy, and Australia [2-4]. There were no differences in serum $\mathrm{pH}$ and bicarbonate levels, rates of severe DKA, and rates of ICU admissions between the two study periods. These findings suggest that children with newly diagnosed T1D were perhaps less influenced by the pandemic.

We found that during lockdown, nearly 70\% of patients with the newly diagnosed T1D and nearly $70 \%$ of the patients with established T1D presented with DKA; however, the small sample size limits interpretation of these findings and suggests the need for further study.

The current study has some limitations. First, we investigated only the first 3 months of the SARS-CoV-2 pandemic. Our results, therefore, may not apply to the later periods of the pandemic. Second, because of the retrospective cross-sectional design of the study, conclusions of a causal nature cannot be drawn from the results. Third, since DKA prevalence at T1D 
diagnosis varied across countries, the findings of this study may not be generalizable to other populations [1].

\section{CONCLUSIONS}

Study findings provide evidence for the increased rates of DKA in children with established T1D during the first months of the pandemic in Israel. The findings suggest that the severity of DKA at ED presentation in children with T1D was not influenced by the pandemic.

Since the pandemic is still progressing, it is important to enhance patient and clinician awareness of this phenomenon while simultaneously exploring possible causes such as delayed ED presentation or suboptimal followup care during the pandemic.

\section{ACKNOWLEDGEMENTS}

Funding. This research did not receive any specific grant from funding agencies in the public, commercial, or not-for-profit sectors. The sponsor for the journal's Rapid Service Fee is Rambam Health Care Campus.

Authorship. All named authors meet the International Committee of Medical Journal Editors (ICMJE) criteria for authorship for this article, take responsibility for the integrity of the work as a whole, and have given their approval for this version to be published.

Authorship Contributions. Dr Jacob designed the study, analyzed and interpreted the data, reviewed the literature, and critically revised the article. Dr Weiser designed the study, analyzed and interpreted the data, reviewed the literature, and critically revised the article. Giora Weiser has equal contribution as first author. Drs Krupik, Takagi, Peled, Pines, Hashavya, Gur-Soferman, Gamsu, Kaplan, Mimon, Oren, and Padeh analyzed and interpreted the data, carried out the initial analysis and critically revised the article. Dr Shavit conceived the idea for the study, analyzed and interpreted the data, and drafted the manuscript. Dr Shavit has full access to all the data in the study and takes responsibility for the integrity of the data and the accuracy of the data analysis. All authors approved the final manuscript as submitted and agree to be accountable for all aspects of the work.

Disclosures. Ron Jacob, Giora Weiser, Danna Krupik, Dania Takagi, Shuny Peled, Naama Pines, Saar Hashavya, Hagar Gur-Soferman, Shirly Gamsu, Or Kaplan, Michal Maimon, Shahar Oren, Gabi Padeh and Itai Shavit declare they have nothing to disclose.

Compliance with Ethics Guidelines. The study was approved by the institutional review boards of all participating hospitals: Rambam Health Care Campus, Ha'Emek Medical Center, Shaare Zedek Medical Center, Ziv Medical Center, Meir Medical Center, Hadassah Medical Center, Samson Assuta Ashdod University Hospital, Shamir Medical Center, Soroka Medical Center, Kaplan Medical Center, and Schneider Children's Medical Center. The study was performed in accordance with the Helsinki Declaration of 1964 and its later amendments. Informed consent was not required because of the retrospective nature of the study.

Data Availability. The datasets generated during and/or analyzed during the current study are available from the corresponding author on reasonable request.

Open Access. This article is licensed under a Creative Commons Attribution-NonCommercial 4.0 International License, which permits any non-commercial use, sharing, adaptation, distribution and reproduction in any medium or format, as long as you give appropriate credit to the original author(s) and the source, provide a link to the Creative Commons licence, and indicate if changes were made. The images or other third party material in this article are included in the article's Creative Commons licence, unless indicated otherwise in a credit line to the material. If material is not included in the article's Creative Commons licence and your intended use is not permitted by statutory 
regulation or exceeds the permitted use, you will need to obtain permission directly from the copyright holder. To view a copy of this licence, visit http://creativecommons.org/licenses/by$\mathrm{nc} / 4.0 /$.

\section{REFERENCES}

1. Cherubini V, Grimsmann JM, Åkesson K, et al. Temporal trends in diabetic ketoacidosis at diagnosis of paediatric type 1 diabetes between 2006 and 2016: results from 13 countries in three continents. Diabetologia. 2020;63(8):1530-41.

2. Kamrath C, Mönkemöller K, Biester T, et al. Ketoacidosis in children and adolescents with newly diagnosed type 1 diabetes during the COVID-19 pandemic in Germany. JAMA. 2020;324(8):801-4.

3. Rabbone I, Schiaffini R, Cherubini V, et al. Has COVID-19 delayed the diagnosis and worsened the presentation of type 1 diabetes in children? Diabetes Care. 2020. https://doi.org/10.2337/dc20-1321

4. Lawrence C, Seckold R, Smart C, et al. Increased paediatric presentations of severe diabetic ketoacidosis in an Australian tertiary center during the COVID19 pandemic. Diabet Med. 2020. https://doi.org/10. 1111/dme.14417.

5. Kaji AH, Schriger D, Green S. Looking through the retrospectoscope: reducing bias in emergency medicine chart review studies. Ann Emerg Med. 2014;64(3):292-8.

6. Wolfsdorf J, Glaser N, Sperling MA, American Diabetes Association. Diabetic ketoacidosis in infants, children, and adolescents: a consensus statement from the American Diabetes Association. Diabetes Care. 2006;29(5):1150-9.

7. Isba R, Edge R, Jenner R, Broughton E, Francis $\mathrm{N}$, Butler J. Where have all the children gone? Decreases in paediatric emergency department attendances at the start of the COVID-19 pandemic of 2020. Arch Dis Child. 2020;105(7):704. 